BIOMEDICA

Vol. 2, No. 1 - 1982

ACTUALIZACIONES

\title{
VACUNA CON POLISACARIDOS DEL NEUMOCOCO
}

\author{
RECOMENDACIONES DEL COMITE ASESOR SOBRE PRACTICAS DE INMUNIZACION \\ ( ACIP) CDC. ATLANTA U.S.A.
}

\section{INTRODUCCION}

En 1977 se licenció en Estados Unidos la vacuna polivalente, de polisacáridos, contra la enfermedad producida por el neumococo (Streptococcus pneumoniae). Este documento incluye un resumen de lo conocido sobre la vacuna y una guía para su uso en personas y grupos seleccionados.

\section{ENFERMEDAD NEUMOCOCICA} PREVENIBLE POR VACUNA

No se tienen datos sobre la exacta ocurrencia de la enfermedad neumocócica grave en los Estados Unidos. La estimación del problema se basa en encuestas limitadas, reportes de algunas investigaciones y varios estudios comunitarios (Tabla 1).

Los estudios comunitarios indican que la neumonía por neumococo representa, por lo general, menos del $25 \%$ de los casos de neumonía. No obstante, debe considerarse como un problema importante, aún en esta era de los antibióticos, por la inminente cantidad de casos y muertes que ocurren anualmente.

La neumonía por neumococo se presenta en todos los grupos de edad, aumentando su incidencia con la edad, principalmente por encima de los 40 años. La meningitis por neumococo se ve principalmente en las edades menores, en particular en el grupo de dos o menos años de edad. La mortalidad por enfermedad neumocócica es mayor en pacientes con bacteremia o meningitis, en pacientes en condiciones de salud deficiente y en personas de edad avanzada.

Los pacientes con ciertos estados crónicos están claramente en mayor riesgo de desarrollar una infección neumocócica y de experimentar enfermedades neumocócicas más severas. Estas condiciones incluyen: anemia de células falciformes (Sickle-cells), mieloma múltiple, cirrosis, insuficiencia renal, disfunsión esplénica, esplenectomía y transplantes de órganos. Otros individuos que están en gran riesgo de desarrollar infección por neumococo y de hacer enfermedades severas, son los alcohólicos o quienes sufren de diabetes mellitus, insuficiencia cardíaca congestiva, enfermedad pulmonar crónica o condiciones asociadas con la inmunosupresión. Los pacientes que sufren pérdidas de líquido cefalorraquídeo como consecuencia de fracturas de cráneo o de procedimientos neuroquirúrgicos pueden hacer meningitis neumocócicas recurrentes.

La vigilancia aplicada sobre la susceptibilidad de las cepas de $S$. pneumoniae, recientemente aisladas, a los antibióticos, no ha mostrado que haya una tendencia hacia el aumento de la resistencia a la penicilina. Menos del 2\% de los aislamientos de $S$. pneumoniae, clínicamente significantes, hechos de 1978 a 1980 , fueron relativamente resistentes a la penicilina (concentración mínima inhibitoria de 0,1 a 0,9 microgramos por ml). La penicilina continúa siendo el agente antimicrobiano de elección para tratar la enfermedad neumocócica invasiva.

\footnotetext{
* Traducción autorizada por el COC., del artículo publicado en: Morbidity and Mortality Weekly Report. $1981 ; 30(33) ; 410$.
} 


\section{VACUNA CON EL POLISACARIDO DEL NEUMOCOCO}

La vacuna neumocócica licenciada en 1977 para ser usada en los Estados Unidos contienen el material capsular purificado, de 14 "tipos" de S. pneumoniae (Tipos daneses: 1, 2, 3, 4, 6A, 7F, 8, 9N, 12F, 14, 18C, $19 F, 23 F$ y 25). Cuando se inició la preparación de la vacuna se extraían los polisacáridos por separado y se combinaban en un producto final. Cada dosis de vacuna contiene 50 microgramos de cada uno de los polisacáridos. Los 14 "tipos" de bacterias que están representadas en la vacuna son responsable del $68 \%$ de los casos de enfermedad bacterémica por neumococo en los Estados Unidos (3) y un 17\% de los casos son debidos a serotipos inmunológicamente relacionados con los "tipos" de neumococo de la vacuna. Los estudios hechos sobre reactividad cruzada entre los anticuerpos humanos contra "tipos" relacionados sugieren la posibilidad de que ocurra la protección cruzada entre algunos de tales "tipos", (por ejemplo, entre 6A y 6B) (4).

La mayoría de los adultos sanos responden a la vacuna y en dos a tres semanas presentan un aumento al duplo de los anticuerpos tipo-específicos medidos por radio-inmunoensayo. No se han determinado los títulos de anticuerpos para cada serotipo.

\section{EFECTIVIDAD DE LAS VACUNAS CON EL POLISACARIDO DEL NEUMOCOCO}

En las décadas de los años 1920, 1930 y 1940 se desarrollaron y probaron varias vacunas neumocócicas. De 1937 a 1943 se hizo en un ancianato la prueba (no ciega) de una vacuna trivalente (5), con la cual se demostró protección contra neumonía y bacteremia debidas a los "tipos" de neumococo empleados en la vacuna y a otros no presentes en ella. En 1944 se probó una vacuna tetravalente, con polisacáridos, en una población militar jóven, con alta tasa de endemicidad de la enfermedad, lográndose la prevención de la neumonía producida por los "tipos" empleados en esta vacuna (6) más no de la producida por otros "tipos". Entre 1945 y 1947 se distribuyó en los Estados Unidos una vacuna de combinación del polisacárido de varios neumococos, pero, cuando se comenzaron a conseguir antibióticos efectivos, se fue abandonando su uso y los productores descontinuaron voluntariamente la producción.

En la década de 1970 se hizo en Sudáfrica una prueba de campo con una vacuna dodecavalente de neumococo en una población de mineros del oro, adultos jóvenes, sanos, en quienes había una alta incidencia de neumonía neumocócica (200 casos/1000 personas/año) (7). Esta vacuna confirió protección tipo-específica y redujo significativamente la frecuencia de la neumonía neumocócica y de la morbilidad respiratoria general. En una población nativa de Nueva Guinéa, donde se presentaban gran cantidad de casos de enfermedades respiratorias agudas y crónicas causadas en su mayoría por neumococo, se redujeron significativamente la morbilidad y la mortalidad por neumonía, cuando se probó una vacuna de 14 serotipos (8).

En los Estados Unidos se llevaron a cabo dos experiencias, al azar, controladas, aplicando vacuna neumocócica en adultos de edad avanzada (9). Una de ellas en pacientes ambulatorios mayores de 45 años y la otra en pacientes siquiátricos crónicos recluídos en una casa de reposo. En ninguno de los dos estudios hubo diferencia alguna en la ocurrencia de morbilidad y mortalidad respiratorias entre el grupo que recibió vacuna polivalente de neumococos y el que recibió un placebo. En el primer estudio los datos sugirieron protección contra la enfermedad bacterémica por neumococo, pero la incidencia de enfermedad neumocócica fue tan baja (menos de 2,5 casos por 1.000 personas, por año) que no dio bases para afirmar la eficacia de la vacuna. En el otro grupo en estudio no hubo menos casos de neumonía, diagnosticados radiológicamente, en los vacunados que en el grupo control.

Los datos de estos dos estudios se analizaron definiendo los casos según la seroconversión a un serotipo de la vacuna y la documentación radiográfica de la neumonía. Con esta definición se calculó que la eficacia de la vacuna osciló entre el 80 y el $100 \%$. Sin embargo, como los vacunados no mostraron un aumento de los títulos de anticuerpos después de ser revacunados, tales vacuna- 
VACUNA CON POLISACARIDOS DEL NEUMOCOCO

TABLA 1

Ocurrencia Estimada de Enfermedad Neumocócica Seria en los Estados Unidos

\begin{tabular}{|c|c|c|c|}
\hline $\begin{array}{l}\text { Enfermedad } \\
\text { Neumocócica }\end{array}$ & $\begin{array}{l}\text { Casos Estimados } \\
\text { (Miles por año) }\end{array}$ & $\begin{array}{l}\text { Incidencia Estimada } \\
\text { Tasa anual| } 100.000\end{array}$ & $\begin{array}{l}\text { Tasa de } \\
\text { letalidad } \%\end{array}$ \\
\hline Neumonía & 150 a 570 & 68 a 260 & 5 a 7 \\
\hline Meningítis (1) & 2,6 a 6,2 & 1,8 a 2,8 & 32 \\
\hline Bacteremia (2) & 16 a 55 & 7 a & 20 \\
\hline
\end{tabular}

dos no estarían habilitados para hacer una seroconversión ante una infección natural y por lo tanto se dificulta la documentación de casos en ellos. La eficacia de la vacuna, medida por esta definición de casos, parecería entonces haberse sobrestimado.

Han sido muy pocos los estudios sobre la eficacia de la vacuna neumocócica en los niños. En general se ha visto que esta vacuna es menos antigénica para los niños menores de 2 años que lo son otras vacunas. Sin embargo, en un pequeño estudio, no sometido al azar, efectuado en una población de niños y adultos jóvenes ( 2 a 25 años de edad) que padecían de anemia de células falciformes (Sickle cells) o habían sido esplenectomizados, se encontró una significativa reducción en la ocurrencia de enfermedad bacterémica a neumococo, por la inmunización con una vacuna octavalente (10).

Recientemente se ha propuesto un método para evaluar la protección lograda por las vacunas neumocócicas, que consiste en comparar la distribución de los serotipos de neumococo aislados de la sangre o del líquido cefalorraquídeo de pacientes vacunados y no vacunados (11). Cuando este método se empleó, comparando 36 pacientes mayores de 10 años que se vacunaron sin haber sido clasificados en cuanto a sus condiciones clínicas basales, con un grupo control de similares condiciones pero no vacunado y de tamaño diez veces mayor, se encontró una tasa del $49 \%$ en la efectividad de la vacuna $\left(66^{\%} \%\right.$ cuando se consideraron únicamente los individuos de los cuales se hizo el aislamiento en sangre). Gracias a que la mayoría de las personas se prestaron para la evaluación, se pudieron establecer los estimativos para fijar categorías diagnósticas específicas y se redujeron los amplios intervalos de confidencia para el análisis.

Se desconoce la duración de la protección inducida por la vacuna, auncuando sí se están llevando a cabo estudios sobre la persistencia de títulos elevados de anticuerpos; los datos corrientemente informados muestran títulos elevados entre 3 y 5 años después de la vacunación.

\section{EFECTOS COLATERALES Y REACCIONES ADVERSAS}

Cerca de la mitad de quienes reciben vacuna de neumococos padecen efectos colaterales, como eritema y moderado dolor en el sitio de la inoculación.

Los efectos adversos severos, tipo reacción anafiláctica, han sido bastante raros; del orden de 5 por un millón de dosis administradas.

Las reacciones sistémicas y locales, severas, han sido comunes entre los adultos que reciben una segunda dosis (12). Se piensa que son la resultante de reacciones locales antigeno-anticuerpo, en las cuales están comprometidos los anticuerpos inducidos por la vacuna previa. Se desconoce si una iiffección previa por uno de los serotipos de $S$ 
pneumoniae presentes en la vacuna pueden desencadenar una reacción local comparable, después de la vacunación. Varios estudios indican que se pueden administrar simultáneamente, en sitios diferentes, vacuna neumocócica y vacuna de influenza, sin que se aumenten los efectos colaterales (13), pero, debe hacerse énfasis en que en el adulto la vacuna neumocócica solo puede ser administrada una vez. Los datos de revacunación en el niño no son suficientes como base para comentarios al respecto.

\section{USO DE LA VACUNA}

Se ha demostrado, en poblaciones seleccionadas, que la vacuna neumocócica de 14 serotipos conseguida corrientemente (al igual que las primeras producidas) reduce en aproximadamente un $80 \%$ la neumonía con bacteremia causada por los tipos de S. pneumoniae empleados en ella. Para extrapolar esta información y recomendar el uso de la vacuna es conveniente reconocer que los datos de efectividad provienen primordialmente de estudios adelantados en grupos de adultos expuestos a un alto riesgo de enfermar pero que no estaban crónicamente enfermos. Como la edad y algunas enfermedades crónicas aparentemente predisponen a los individuos para hacer enfermedad neumocócica más severa, sería ideal que las recomendaciones sobre inmunización pudieran basarse en investigaciones clínicas prolijas, hechas en grupos de pacientes jóvenes y de pacientes con enfermedades crónicas. No obstante que continúan acumulándose datos sobre la efectividad de la vacuna neumocócica en personas con enfermedad crónica y en otras, no son suficientes todavía para interpretaciones concluyentes. Por tanto, las siguientes recomendaciones del Comité se derivan de datos que se admiten como limitados:

1. Con base en las evidencias preliminares, las personas mayores de dos años, que padezcan disfunción esplénica o anesplenia anatómica, pueden beneficiarse con la vacunación. Auncuando se han comunicado fallas de la vacuna, debidas quizas a perturbaciones en la respuesta inmune, se recomienda de toda forma su administración en estos pacientes porque están en alto riesgo de desarrollar bacteremias fatales.

2. Deben vacunarse los adultos y los niños mayores de dos años, con enfermedades crónicas y en riesgo inminente o posible de padecer una enfermedad neumocócica o sus complicaciones (véase atrás). La vacuna aumentaría su beneficio a medida que los pacientes avanzan en edad por cuanto los menores tienen mayor riesgo de morir de infecciones neumocócicas. La eficacia de la vacuna en estos grupos requiere mayor evaluación y continúa siendo estudiada.

3. En algunos grupos de población, como en guarderías y otras instituciones cerradas, existe un mayor riesgo de que se presenten brotes epidémicos o tasas elevadas de endemia de enfermedad neumocócica con características de severidad. Bajo estas. circunstancias debe considerarse la vacunación global de las comunidades cerradas.

4. Puede ocurrir, auncuando por rareza, que en la población general se presenten brotes epidémicos, localizados, de enfermedad neumocócica producida por "tipos" empleados en la vacuna; en tal caso debe considerarse la inmunización selectiva de aquellas personas en alto riesgo.

5. No se tienen aún suficientes datos para recomendar el empleo rutinario de la vacuna neumocócica en programas de inmunización para la población general, incluídos los menores.

\section{PRECAUCIONES}

No se ha evaluado la inocuidad de la vacuna neumocócica en la mujer gestante; por lo tanto no debe ser usada durante el embarazo, al menos que el riesgo de infección esté sustancialmente aumentado.

Debido al marcado incremento de las reacciones adversas frente a las reinoculaciones con vacuna neumocócica, no deben aplicarse segundas dosis o de refuerzo. El registro de todos los eventos de vacunación facilitará el que no se apliquen dosis repetidas. 
VACUNA CON POLISACARIDOS DEL NEUMOCOCO

\section{BIBLIOGRAFIA}

1. Fraser DW, et al. Risk factors in bacterial meningitis: Charleston County, South Carolina. J. Infect Dis 1973; $127: 271$.

2. Filice GA, etal. Pneumococcal bacteremia in Charleston County, Sounty Carolina. Am J Epidemiol 1980; 112: 828 .

3. Broome CV, Facklam RR. Epidemiology of clinical significant isolates of Streptococcus pneumoniae in the United States. Review of infectious Diseases 1981; 3: 277 .

4. Robbins $J B$, et al. Comparative immunogenicity of group 6 pneumocoocal type $6 \mathrm{~A}$ (6) and type $6 \mathrm{~B}$ (26) capsular polysaccharides, Infect Immun 1979; 26: 1116.

5. Kaufman P. Pneumonia in old age. Active immunization against pneumonia with pneumonococcus polysaccharide; results of 6-year study. Arch Intern Med 1947; 79: 518 .

6. Macleod CM, et al. Prevention of pneumococcal pneumonia by immunization with specific capsular polysaccharides. J. Exp Med 1954; 82: 445 .

7. Austrian R, et al. Prevention of pneumococcal pneumonia by vaccination. Trans Assoc Am Physicians 1976; 89: 184
8. Riley ID, et al. Immunisation with a polyvalent pneumococcal vaccine: reduction of adult respiratory mortafity in a New Guinea Highlands cominity. Lancet $1977 ; 1: 1338$.

9. Austrian R. Surveillance of pneumococcal infection for field trials of polyvalent pneumococcal vaccines. Report DAB.VDP-12-84, National Intitutes of Health, 1980.

10. Ammann AJ, et al. Polyvalent pnemococcal polysaccha ride immunization of patients with sickle call anemia and patients with splenectomy. N. Engl J Med 1977; 297: 897.

11. Broome CV, et al. Pneumococcal disease after pneumoco ccal vaccination: an alternative method to estimate the efficacy of pneumococcal vaccine. N. Engl J Med 1980; 303: 549 .

12. Borgoño $J M$, et al. Vaccination and revaccination with polyvalent pneumococcal polysaccharide vaccines in adults and infants (40010). Proc. Soc Exp Biol Med 1978; 157: 148 .

13. Mufson MA, etal. Polyvalent pneumococcal vaccine given alone and in combination with bivalent influenza virus vaccine (40804). Proc Soc Exp Biol Med 1980; 163: 498 